\title{
Measurement of associated production of vector bosons with b-jets, with a charm quark, and with a $J / \psi$ meson in ATLAS
}

Miriam WATSON*, on behalf of the ATLAS Collaboration

University of Birmingham

E-mail: Miriam.Watsonecern.ch

\begin{abstract}
Recent results on heavy flavour production from the ATLAS experiment are presented, using proton-proton collisions at $\sqrt{s}=7 \mathrm{TeV}$ recorded at the Large Hadron Collider. These results involve the production of vector bosons in conjunction with heavy flavour quarks, and allow QCD predictions to be tested. Measurements of $W$ and $Z$ bosons with $b$-jets and single $c$-quark jets probe the parton density of the proton, while the associated production of vector bosons with $J / \psi$ mesons can be compared to quarkonium production models.
\end{abstract}

The 15th International Conference on B-Physics at Frontier Machines at the University of Edinburgh, 14 -18 July, 2014

University of Edinburgh, UK

${ }^{*}$ Speaker. 


\section{Introduction}

The predictions of quantum chromodynamics (QCD) can be tested in a new regime at hadron colliders, through the study of heavy quarks produced in conjunction with vector bosons. These final states occur in measurements of Higgs boson decays and in searches for physics beyond the Standard Model, and it is therefore vital to understand such processes. In addition to testing $\mathrm{QCD}$, vector bosons produced with bottom or charm quarks give a unique sensitivity to the heavy quark density of the proton. Measurements of the associated production of vector bosons with quarkonium states (e.g. $J / \psi$ ) can provide insight into the quarkonium production mechanism in a new environment.

The analyses presented here are based on the data recorded in the ATLAS detector [1] at the LHC in 2011 at $\sqrt{s}=7 \mathrm{TeV}$, with an integrated luminosity of $4.6 \mathrm{fb}^{-1}$. Samples of $Z$ or $W$ bosons are selected in their leptonic final states, using muons or electrons. These leptons give clean signatures for triggering and for particle identification. Events containing $Z$ bosons are identified using pairs of oppositely charged leptons, with an invariant mass close to the $Z$ mass, while $W$ boson decays are selected through the combination of a single lepton and a significant missing transverse energy from the neutrino. Multi-jet backgrounds are suppressed by requiring the leptons to be isolated, using either tracking or calorimeter information around the lepton.

\section{2. $W$ boson production in association with $b$-jets}

The cross-section for $W$ boson production in association with $b$-quark jets has been measured for one-jet and two-jet final states [2]. This channel is an important background for the Higgs production process $W H(\rightarrow b \bar{b})$ and in searches for new physics. The $b$-jet contribution is extracted from a template fit to weights from a b-tagging algorithm, and corrected for backgrounds and detector effects. Figure 1 shows the fiducial $W+b$-jets cross-sections, which are compared with nextto-leading order predictions from MCFM [3] and POWHEG [4], and leading-order ALPGEN [5] predictions. The one-jet data lie slightly above the predictions, but are consistent within $1.5 \sigma$, while the two-jet measurements agree well with the predictions. Figure 2 shows the differential cross-section as a function of the leading $b$-jet $p_{\mathrm{T}}$. The predictions are found to underestimate the one-jet data slightly at high $p_{\mathrm{T}}$.

\section{3. $W$ boson production in association with charm}

The production of a $W$ boson in association with a single charm quark has also been studied [6]. To leading order, this process is dominated by the $g_{s}$ and $g \bar{s}$ production channels, at the level of $90 \%$, and is therefore an excellent probe of the $s$-quark distribution function in the proton. Previous analyses have shown a preference for $s$-quark suppression relative to $d$-quarks in the sea; however, a recent analysis of ATLAS $W$ and $Z$ production and HERA DIS data [7] favoured a flavoursymmetric sea at LHC scales and Bjorken $x \sim 0.01$. The $W+$ charm data allow these properties to be tested further. 


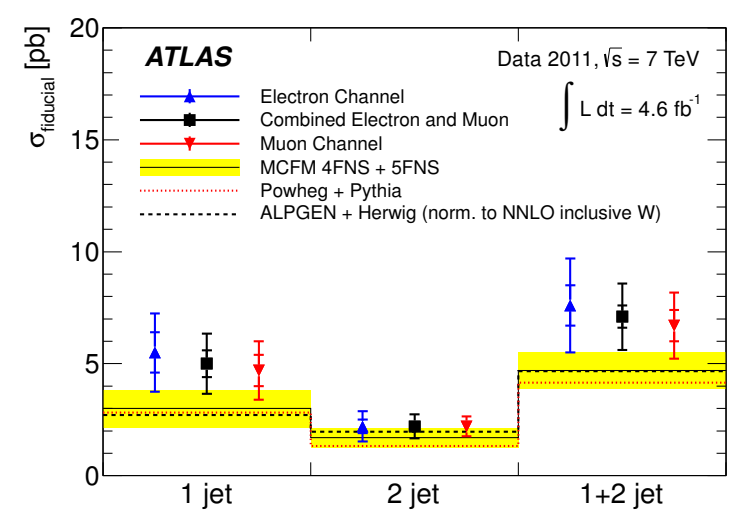

Figure 1: $\quad W+b$-jet cross-sections in the 1-jet, 2 -jet and 1+2-jet fiducial regions [2].

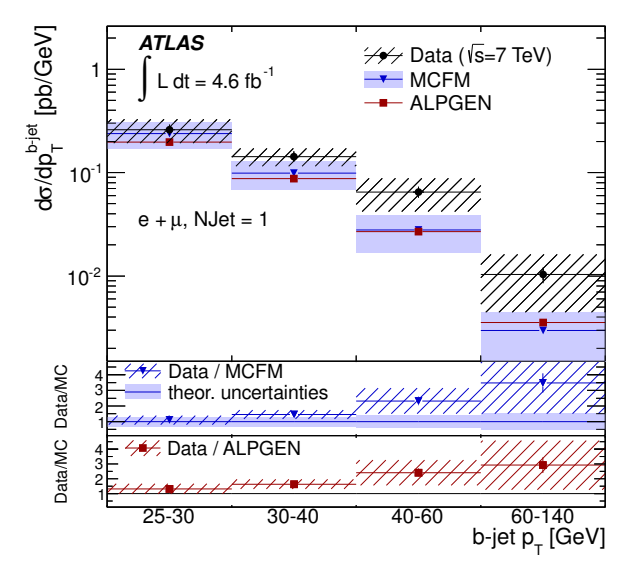

Figure 2: Differential $W+b$-jet cross-section as a function of $b$-jet $p_{\mathrm{T}}$ in the 1-jet fiducial region [2].

Charm quarks are tagged either using charmed hadrons in the decay modes $D^{+} \rightarrow K^{-} \pi^{+} \pi^{+}$ and $D^{*+} \rightarrow D^{0} \pi^{+}$(and charge conjugates), or by requiring a particle jet containing a soft semileptonic decay to a muon. For the signal process, the charges of the $W$ boson and the charm quark are expected to be opposite in sign. Since most background processes are charge-symmetric, for example $W+b \bar{b}$ or $W+c \bar{c}$, signal distributions can be extracted by subtracting same-sign from oppositesign distributions.

The $W+$ charm cross-sections are measured within fiducial phase space regions, and are compared with predictions from the next-to-leading order aMC@NLO [8] in combination with HERWIG++ [9] and a number of different PDF sets. These NLO and NNLO PDF sets give rise to variations of approximately $25 \%$ in the predicted cross-sections. The uncertainties on the data cover a wide range of predictions, but tend to disfavour those which have a suppressed $s$-quark component (MSTW2008, NNPDF2.3 and HERAPDF1.5), as shown in Figure 3 for the $W^{+}+\bar{c}$-jet cross-section. The ratio of the $s$-to- $d$ sea-quark distributions is evaluated in the context of HERAPDF1.5 as $0.96_{-0.30}^{+0.26}$ at $Q^{2}=1.9 \mathrm{GeV}^{2}$. This is consistent with an SU(3)-symmetric light-quark sea. The ratio of cross-sections for the final state $W^{+}+\bar{c}$ relative to $W^{-}+c$ is also studied, and is shown in Figure 4 for $W+\mathrm{D}^{(*)}$. At the precision of the current measurement, the data are consistent within uncertainties with PDFs which include either a small $s: \bar{s}$ asymmetry or no asymmetry.

\section{4. $Z$ boson production in association with $b$-jets}

A recent analysis of the production of $Z$ bosons in association with $b$-jets [10] allows several aspects of QCD predictions to be tested. For example, the four- and five-flavour number schemes (4FNS, 5FNS) can be compared, where the 5FNS includes $b$-quark density in the proton, as can predictions involving massive or massless $b$-quarks, or calculations to different orders. The $b$-jets are identified using template fits to appropriate $b$-tagging variables in either the one- $b$-tag or two- $b$ tag cases, then yields and differential distributions are corrected for reconstruction efficiencies and detector resolution effects. 


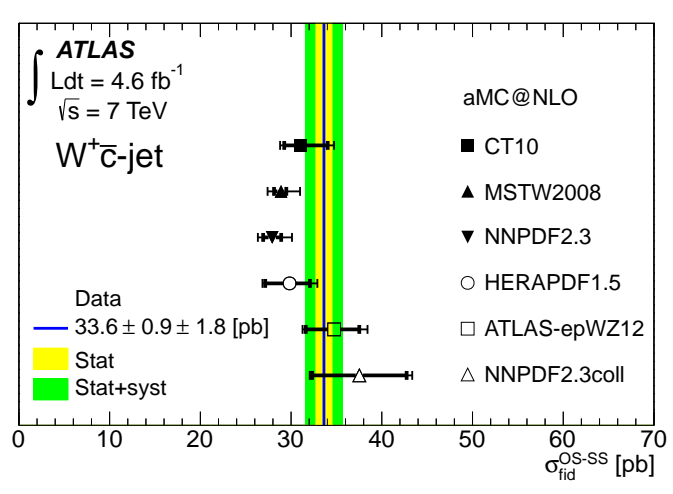

Figure 3: Measured $W^{+}+\bar{c}$-jet fiducial crosssection compared to various PDF predictions based on aMC@NLO [6].

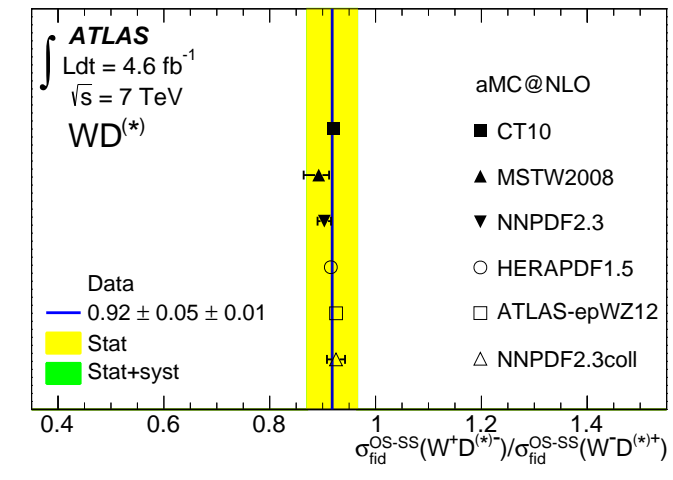

Figure 4: Measured fiducial cross-section ratio for $\left(W^{+}+\mathrm{D}^{(*)-}\right) /\left(W^{-}+\mathrm{D}^{(*)+}\right)$, compared to various PDF predictions based on aMC@NLO [6].

Cross-sections for $Z+\geq 1 b$-jet and $Z+\geq 2 b$-jets are compared with various predictions, as shown in Figures 5 and 6, respectively. Firstly, the data are compared with MCFM [3], an NLO prediction in the 5FNS, which is evaluated for several different PDF sets. All the MCFM predictions agree with the data within uncertainties. Two different predictions from aMC@NLO [8] plus HERWIG++ [9] are also considered. One is a $Z+b$ calculation in the 5FNS and the other is $Z+b \bar{b}$ in the $4 \mathrm{FNS}$. In the $1-b$-jet case, both calculations are next-to-leading order and the dominant difference is the number of flavours; the data are found to prefer the 5FNS. For 2- $b$-jets, the $Z+b$ 5FNS calculation is now a leading-order approximation and does not describe the data so well (in contrast to the NLO 4FNS). Leading-order predictions from ALPGEN [5] and SHERPA [11] are also shown. These underestimate the data, but do not include the theoretical uncertainties. Twelve differential cross-section distributions are measured, allowing more stringent tests of the theoretical predictions. For example, Figure 7 shows the 1-b-jet cross-section as a function of the rapidity of the $\mathrm{Z}$ boson, compared to MCFM with various PDF sets. They all show reasonable agreement, within the scale uncertainties. In contrast, the $2-b$-jet cross-section as a function of the opening angle between the $b$ jets (Figure 8) shows some discrepancies between data and predictions at small angles.

\section{5. $W$ boson production with prompt $J / \psi$ mesons}

An alternative measurement, which looks for quarkonium formation instead of $b$ - or $c$-jets, is the associated production of $W$ bosons with prompt $J / \psi$ [12], where the $J / \psi$ and $W$ boson are both identified in their muon decay modes. The measurement probes the quarkonium production mechanism and the possible contributions from colour singlet and colour octet processes. This signature will also be sensitive to multiple parton interactions, in which the $W$ and $J / \psi$ are produced by two pairs of partons from the same proton-proton collision, and the current measurement includes an estimate of the double parton scattering (DPS) contribution.

As with many $J / \psi$ analyses, the invariant mass and pseudo-proper time are fitted simultaneously to extract the prompt $J / \psi$ component. Each prompt candidate then receives a weight in the 


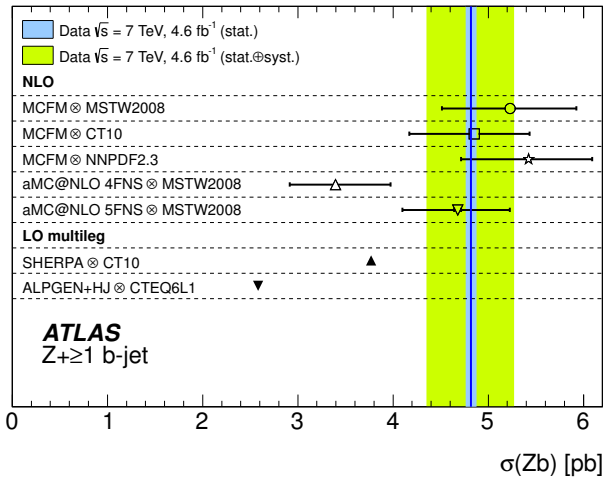

Figure 5: Cross-section for $Z+\geq 1 b$-jet and comparison with theoretical predictions [10].

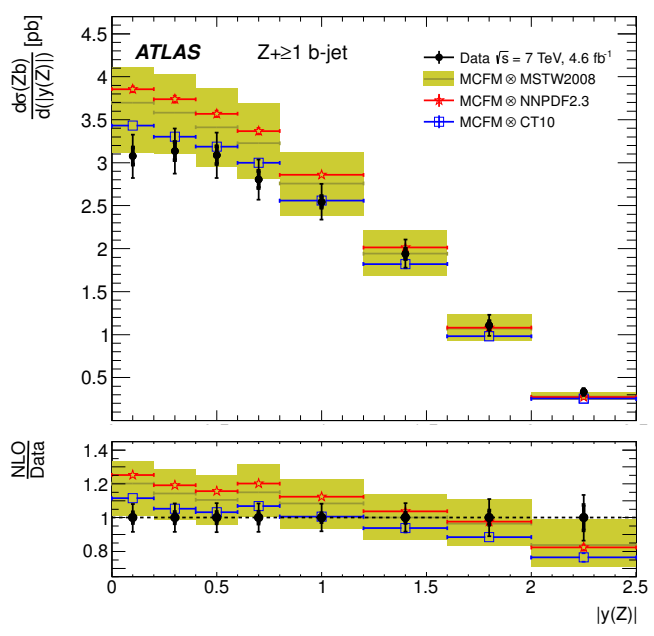

Figure 7: The differential cross-section for $Z+\geq$ $1 b$-jets as a function of the absolute $Z$ boson rapidity, $|y(Z)|[10]$.

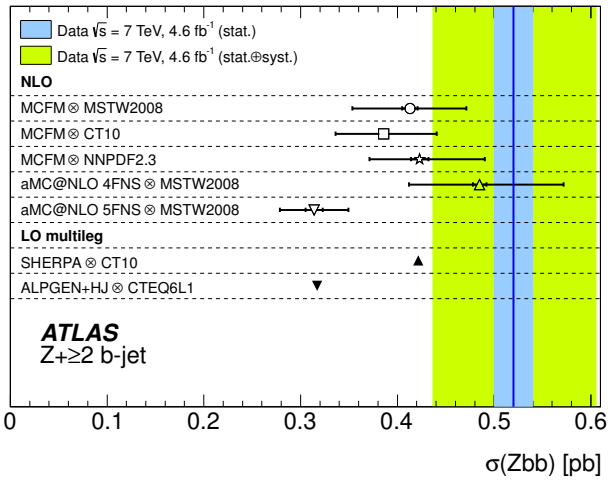

Figure 6: Cross-section for $Z+\geq 2 b$-jet and comparison with theoretical predictions [10].

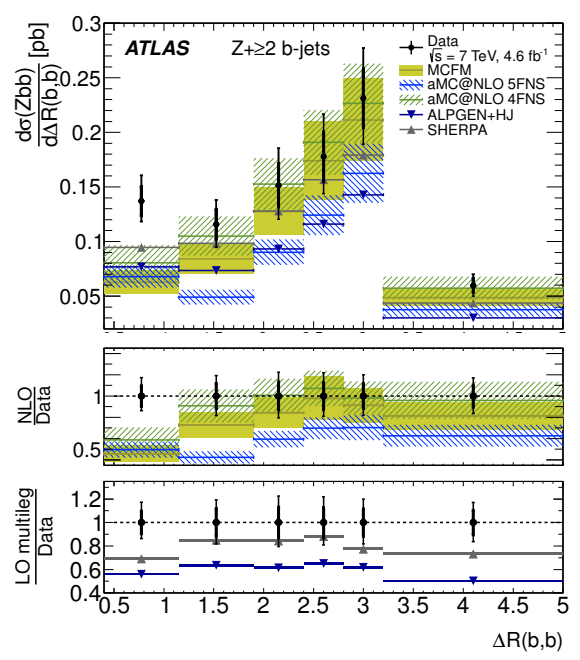

Figure 8: The differential cross-section for $Z+\geq$ $2 b$-jets as a function of the opening angle $\Delta \mathrm{R}(\mathrm{b}$, b) [10].

$W$ transverse mass distribution, which is fitted with $W$ and multi-jet templates, to estimate a multijet contamination of $0.1 \pm 4.6$ events. The associated prompt $J / \psi$ plus $W$ signal is $\sim 27$ events, which is incompatible with the background-only hypothesis at $5.1 \sigma$, and is the first observation of this process.

The cross-section of $W+J / \psi$ is measured relative to the inclusive $W$ cross-section in the same dataset. The contribution from DPS is estimated under the assumption that the $W$ and $J / \psi$ contributions are independent and uncorrelated, and are scaled by the effective cross-section obtained from $W+2$-jet events at ATLAS [13]; its magnitude is $\sim 40 \%$ of the total. The azimuthal angle between the $W$ and the $J / \psi$ will be uniform for independent DPS processes but will peak at $\sim \pi$ for single scattering (SPS) events. The data appear to show both components, as expected, and the differential cross-section as a function of $p_{\mathrm{T}}$ (Figure 9) indicates that SPS dominates at low $p_{\mathrm{T}}$ of 
the $J / \psi$. The cross-section ratio is corrected for $J / \psi$ acceptance and DPS, then the resulting SPS cross-section is compared with predictions from two models: a LO colour singlet model [14] and NLO colour octet model [15]. The theoretical predictions lie below the data, but both are within $2 \sigma$ of the large experimental uncertainties, as shown in Figure 10.

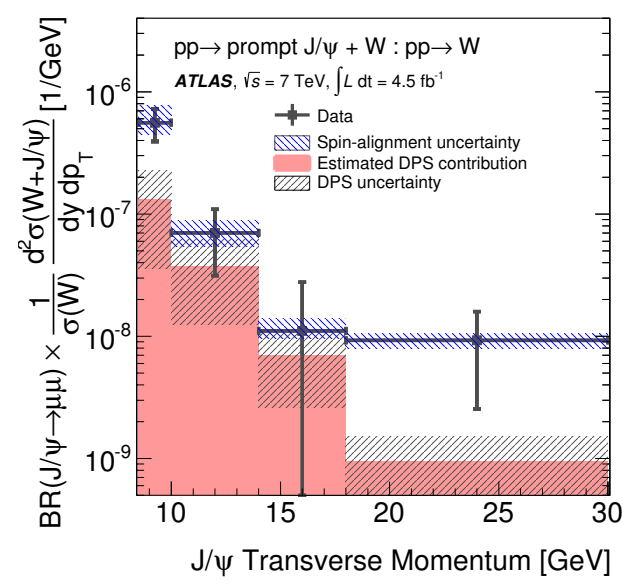

Figure 9: The $W+$ prompt $J / \psi$ to inclusive $W$ production cross-section ratio as a function of $J / \psi p_{\mathrm{T}}[12]$.

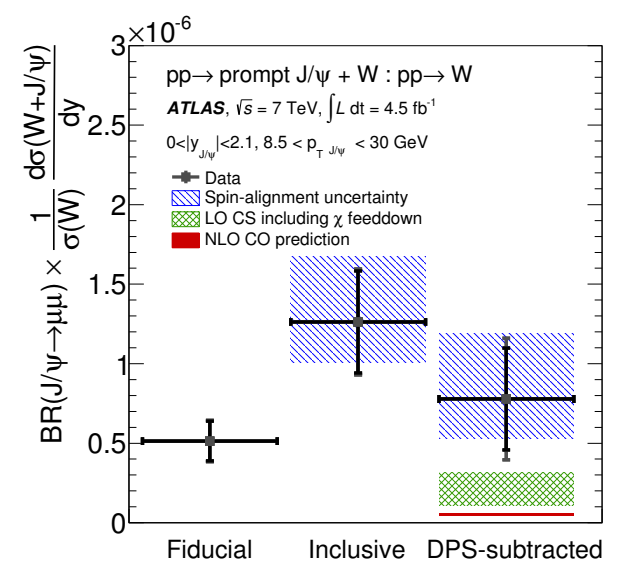

Figure 10: The $W+$ prompt $J / \psi$ to inclusive $W$ production cross-section ratio [12].

\section{Summary}

Several recent measurements of vector boson and heavy flavour production in ATLAS have been presented. These results allow more stringent tests of QCD predictions in a new regime: the measurements with $b$ - or $c$-jets give access to the proton structure function and heavy flavour densities, while measurements of quarkonium in association with a vector boson open a new field in understanding both the mechanism for quarkonium production and the contribution from multiple parton interactions.

\section{Acknowledgments}

The author is supported by a Royal Society Research Fellowship.

\section{References}

[1] ATLAS Collaboration, The ATLAS Experiment at the CERN Large Hadron Collider, JINST 3 (2008) S08003.

[2] ATLAS Collaboration, Measurement of the cross-section for W boson production in association with $b$-jets in pp collisions at $\sqrt{s}=7 \mathrm{TeV}$ with the ATLAS detector, JHEP 06 (2013) 084 [arXiv:1302.2929].

[3] J.M. Campbell and R.K. Ellis, MCFM for the Tevatron and the LHC, Nucl. Phys. Proc. Suppl. 10 (2010) 205-206 [arXiv: 1007 .3492]. 
[4] S. Alioli et al., A general framework for implementing NLO calculations in shower Monte Carlo programs: the POWHEG BOX, JHEP 06 (2010) 043 [arXiv: 1002 . 2581].

[5] M.L. Mangano et al., ALPGEN, a generator for hard multiparton processes in hadronic collisions, JHEP 07 (2003) 001 [hep-ph/ 0206293 ].

[6] ATLAS Collaboration, Measurement of the production of a $W$ boson in association with a charm quark in pp collisions at $\sqrt{s}=7 \mathrm{TeV}$ with the ATLAS detector, JHEP 05 (2014) 068 [arXiv:1402.6263].

[7] ATLAS Collaboration, Determination of the Strange-Quark Density of the Proton from ATLAS Measurements of the $W \rightarrow \ell v$ and $Z \rightarrow \ell \ell$ Cross Sections, Phys. Rev. Lett. 109 (2014) 012001 [arXiv:1203.4051].

[8] J. Alwall et al. The automated computation of tree-level and next-to-leading order differential cross sections, and their matching to parton shower simulations, JHEP 07 (2014) 079

[arXiv:1405.0301].

[9] M. Bahr et al. Herwig++ Physics and Manual, Eur. Phys. J. C 58 (2008) 639 [arXiv: 0803.0883 [hep-ph]].

[10] ATLAS Collaboration, Measurement of differential production cross-sections for a $Z$ boson in association with b-jets in 7 TeV proton-proton collisions with the ATLAS detector, submitted to JHEP [arXiv:1407.3643].

[11] T. Gleisberg et al. Event generation with SHERPA 1.1, JHEP 02 (2009) 007 [arXiv: 0811.4622 [hep-ph]].

[12] ATLAS Collaboration, Measurement of the production cross section of prompt $J / \psi$ mesons in association with a W boson in pp collisions at $\sqrt{s}=7$ TeV with the ATLAS detector, JHEP 04 (2014) 172 [arXiv:1401.2831].

[13] ATLAS Collaboration, Measurement of hard double-parton interactions in $W \rightarrow \ell v+2-j e t$ events at $\sqrt{s}=7$ TeV with the ATLAS detector, New J. Phys. 15 (2013) 033038 [arXiv: 1301.6872].

[14] J. P. Lansberg and C. Lorcé, Reassessing the importance of the colour-singlet contributions to direct $J / \psi+W$ production at the LHC and the Tevatron, Phys. Lett. B 726 (2013) 218-222 [arXiv:1303.5327].

[15] L. Gang et al., QCD corrections to J/ $\psi$ production in association with a $W$-boson at the LHC, Phys. Rev. D83 (2011) 014001 [arXiv: 1012 .3798]. 